Article

\title{
Design and Research of Symmetrical Multi-Throttle Thrust Hydrostatic Bearing Based on Comparative Analysis of Various Meshes
}

\author{
Yongmeng Liu ${ }^{1,2}$, Zifei Cao ${ }^{1,2}$, Yuan Zhang ${ }^{1,2}$, Dawei Wang ${ }^{1,2, *}$, Xiaoming Wang ${ }^{1,2, *}$ and Chuanzhi Sun ${ }^{1,2}$ \\ 1 Center of Ultra-Precision Optoelectronic Instrument Engineering, Harbin Institute of Technology, \\ Harbin 150080, China; lym@hit.edu.cn (Y.L.); 20S001021@stu.hit.edu.cn (Z.C.); \\ 21S001058@stu.hit.edu.cn (Y.Z.); czsun@hit.edu.cn (C.S.) \\ 2 Key Lab of Ultra-Precision Intelligent Instrumentation Engineering, Harbin Institute of Technology, \\ Ministry of Industry and Information Technology, Harbin 150080, China \\ * Correspondence: wdw1989@hit.edu.cn (D.W.); wang_xm@hit.edu.cn (X.W.)
}

check for updates

Citation: Liu, Y.; Cao, Z.; Zhang, Y.; Wang, D.; Wang, X.; Sun, C. Design and Research of Symmetrical Multi-Throttle Thrust Hydrostatic Bearing Based on Comparative Analysis of Various Meshes. Symmetry 2022, 14, 351. https:// doi.org/10.3390/sym14020351

Academic Editors: Sergei

D. Odintsov and Igor V. Andrianov

Received: 26 December 2021

Accepted: 22 January 2022

Published: 10 February 2022

Publisher's Note: MDPI stays neutral with regard to jurisdictional claims in published maps and institutional affiliations.

Copyright: (C) 2022 by the authors. Licensee MDPI, Basel, Switzerland. This article is an open access article distributed under the terms and conditions of the Creative Commons Attribution (CC BY) license (https:// creativecommons.org/licenses/by/ $4.0 /)$.

\begin{abstract}
Due to the advantages of high rotation accuracy and long life, hydrostatic air bearings are widely used in precision rotation equipment, such as machine tools and turntables. It is imperative to reasonably design the structural size of the bearing, especially as the size of the bearing in the precision turntable determines the bearing capacity of the turntable. At present, commercial CFD software is used chiefly for simulation verification for the design of air bearings. The mesh divided in the simulation calculation has a significant impact on the efficiency of the calculation and determines the accuracy of the final simulation results. Therefore, this paper takes the symmetrical multithrottle thrust bearing in the precision turntable as the research object and compares and studies the advantages and disadvantages of sliced structured meshes, continuous structured meshes, and unstructured meshes. On this basis, simulation analysis of bearing capacity for different radial equalizing groove lengths, widths, and depths and explores the influence of the structure size of the thrust bearing on its bearing capacity. The study shows that the length and depth of the throttle groove significantly influence the bearing capacity, while the width has little influence on the bearing capacity. Therefore, under the specific cavity volume to ensure the dynamic characteristics of the bearing, the width of the throttling groove should be reduced first, and the length and depth of the throttling groove should be increased to improve the bearing capacity.
\end{abstract}

Keywords: symmetrical multi-throttle thrust bearing; mesh divide; throttle groove; static characteristics

\section{Introduction}

Ultra-precision processing technology is one of the essential directions for the development of modern advanced manufacturing, and it plays an essential supporting role in the defense industry and civil technology [1]. The primary conditions for achieving ultraprecision processing are processing equipment represented by precision machine tools and inspection equipment represented by precision turntables. The precision spindle system, as the "heart" of such equipment, determines their processing and inspection accuracy [2,3]. In this precision rotating equipment, aerostatic bearings mainly provide a stable rotation reference for the spindle $[4,5]$. While the aerostatic bearings ensure rotation accuracy, their static and dynamic characteristics also determine the spindle's carrying capacity, stiffness, and other properties. Figure 1 shows the UOI series rotor assembly and measurement system developed by the Ultra Precision Photoelectric Instrument Research Institute of Harbin University of technology [6]. The turntable uses a multi-throttle thrust bearing with a symmetrical structure to provide ample bearing capacity and accurate rotation reference. As the measuring object of the system is a large steam turbine rotor, its bearing capacity needs to reach the level of several tons. In order to improve the bearing capacity, a feasible 
scheme is to increase the air floating surface area, but this scheme needs to increase many costs to process larger equipment. For this type of equipment, economy is also an important factor [7]. In contrast, the scheme of improving the bearing capacity by optimizing the structural size of the bearing can not only save the cost but also lighten the design of the measurement system. Therefore, it is necessary to study the influence of bearing structure size on the bearing's static characteristics and then reasonably design the structure and size of the aerostatic bearing to increase the bearing capacity of the turntable and provide better performance for the measurement system.

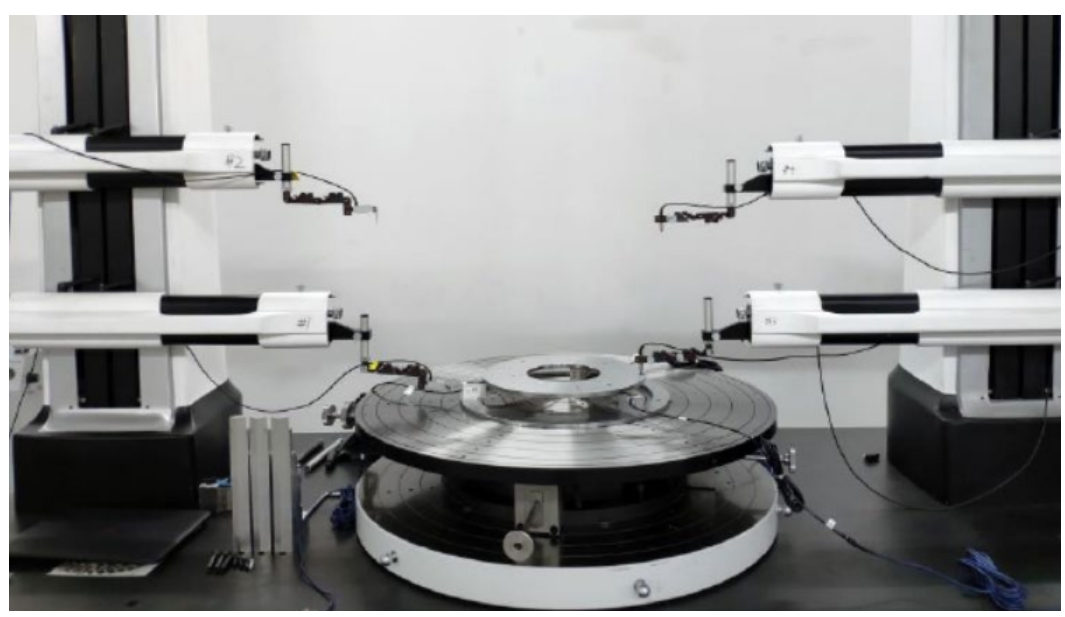

Figure 1. UOI Ultra-precision rotor shape and position error measurement system.

In the middle and late 20th centuries, the development of computational fluid dynamics (CFD) promoted the theoretical analysis and design research of air bearings. At present, CFD software simulation is often used in the design of aerostatic bearings to obtain air film pressure, bearing capacity, gas flow rate, etc. Sahto used CFD software to simulate and solve the internal distribution of pressure of the porous, orifice, and multiple-type aerostatic thrust bearing and analyzed the influence of material and geometric parameters on stiffness and bearing capacity [8]. Yan used CFD software to simulate and analyze the influence of different shapes of pressure-equalizing grooves on bearing performance [9]. Gao used CFD software to simulate and analyze the influence of the herringbone groove on the performance of the aerostatic journal under the conditions of high speed and low pressure [10]. The first step of CFD analysis and calculation is to generate meshes, that is, to subdivide the continuous calculation area in space, divide it into many sub-areas, and determine the nodes in each area. The CFD calculation process's efficiency mainly depends on the generated meshes. The meshes used in the current public research are mainly structured meshes [11-13] and unstructured meshes [14,15], but there is no comparison of the simulation effects of different meshes.

Therefore, this paper takes the multi-throttle thrust bearing with symmetrical structure in the precision air-floating turntable as the research object and compares and studies the advantages and disadvantages of sliced structured grids, continuous structured grids, and unstructured grids. On this basis, simulation analysis of bearing capacity for different radial equalizing groove lengths, widths, and depths discusses the influence of the structure size of the thrust bearing on its bearing capacity. These efforts provide guidance for the simulation and design of compound throttle thrust bearings with periodic symmetry.

\section{Model}

\subsection{Physical Model}

The object studied in this paper is a symmetrical multi-throttle thrust bearing. The bearing comprises orifices and surface equalizing. The overall structure is shown in Figure 2. The bearing comprises orifices, surface-equalizing grooves, and an upper thrust 
plate. Among them, the orifices and the radial, equalizing grooves are symmetrically and cyclically positioned around the circumference. When the bearing is working, the high-pressure gas provided from the outside enters the orifice through the air inlet, and the first throttling effect is generated at the orifice, which is the orifice throttle. Then the gas passes through the circumferential and radial, equalizing grooves on the bearing's surface and flows to the edge of the bearing. The second throttling effect is produced during the flow, which is the surface throttle. The gas finally flows to the atmosphere through the edges on both sides of the inner and outer diameters of the bearing.

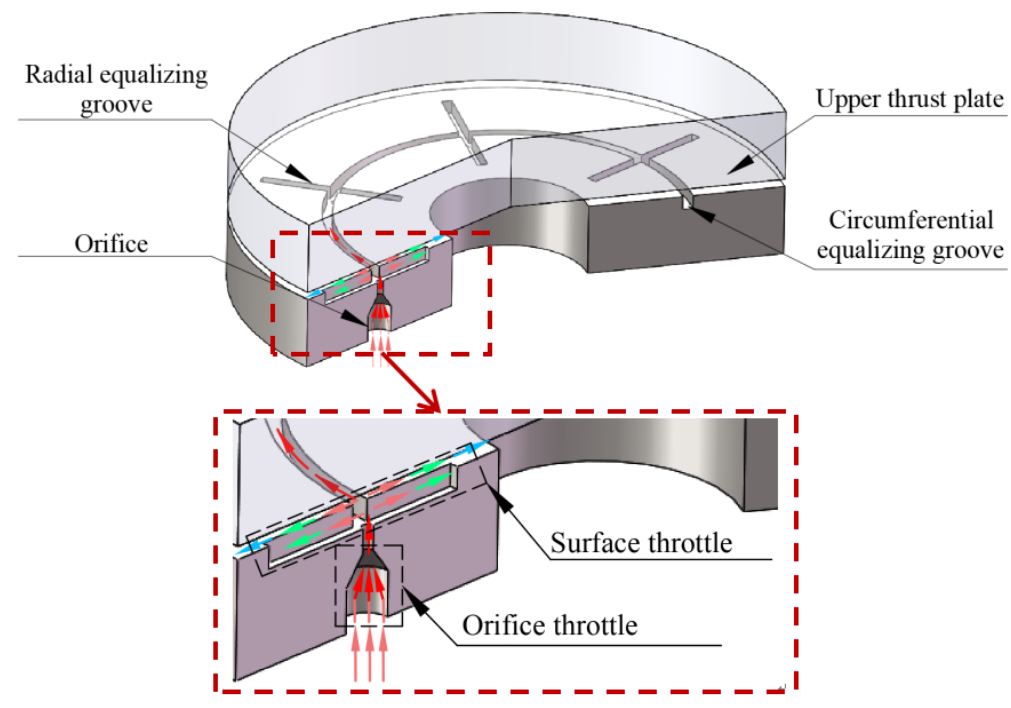

Figure 2. Structure diagram of a symmetrical multi-throttle thrust bearing.

Figure 3 shows the relevant dimensional parameters of the bearing, where $R_{1}$ and $R_{2}$ are the inner and outer diameters of the thrust bearing, respectively. $R_{3}$ is the radius of the distribution circle of the orifice throttle. $L_{\mathrm{g}}$ is the length of the radial equalizing groove. $h_{\mathrm{g}}$ is the depth of the equalizing groove. $b_{\mathrm{g}}$ is the width of the equalizing groove. $h$ is the thickness of the air film. $\mathrm{d}$ is the diameter of the air supply hole.

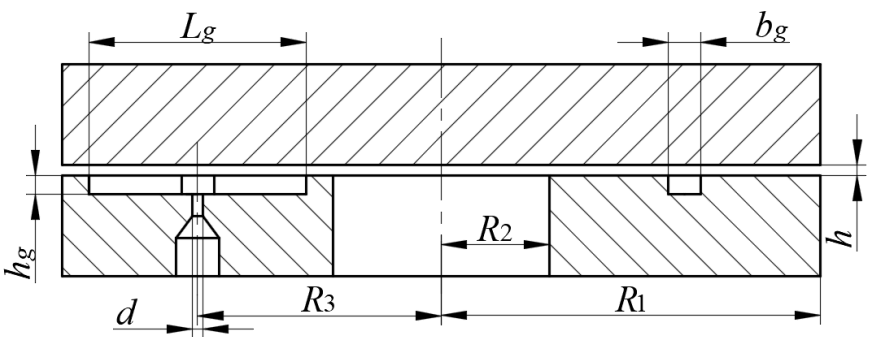

Figure 3. Schematic diagram of dimensional parameters of a bearing.

\subsection{Analysis Model}

Due to the simulation object having a typical symmetrical structure, in order to reduce the number of meshes and improve the efficiency of simulation analysis, the smallest symmetrical part of the gas film is taken, which is one-sixth of the complete model, and establish the three-dimensional analysis model of the gas film [16]. The size parameters used when building the analysis model are: $R_{1}=35 \mathrm{~mm}, R_{2}=10 \mathrm{~mm}, R_{3}=20 \mathrm{~mm}$, $L_{\mathrm{g}}=6 \mathrm{~mm}, h_{\mathrm{g}}=0.1 \mathrm{~mm}, b_{\mathrm{g}}=0.5 \mathrm{~mm}, h=15 \mu \mathrm{m}$ and $d=0.3 \mathrm{~mm}$.

Figure 4 shows the established analysis model and the set boundary. The top surface of the air film at the orifice is the inlet, the inner and outer cylindrical surfaces of the air film are the outlets, the two radial surfaces of the air film are symmetrical planes, and the rest are walls. 


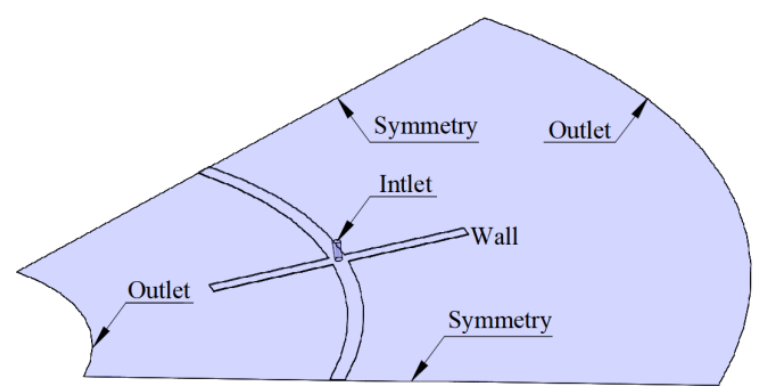

Figure 4. 1/6 Air film model of a symmetrical multi-throttle thrust bearing.

\subsection{Theoretical Model}

Fluid dynamics calculation model can be divided into the turbulence and laminar flow models. According to the parameters of the bearing model studied in this paper, the laminar flow model is selected. The theoretical mathematical model comprises the momentum conservation equation, energy conservation equation, and continuity equation of gas [17].

The momentum conservation equation is shown in Equation (1)

$$
\rho \frac{d \vec{u}}{d t}=\rho F+\nabla P
$$

where $\rho$ is the fluid density, $t$ is time, $\vec{u}$ is the fluid velocity vector, $F$ is the mass force acting on the unit mass of fluid, and $P$ is the stress tensor.

The energy conservation equation is shown in Equation (2)

$$
\rho \frac{d U}{d t}=\rho q+k \nabla^{2} T-\nabla(\vec{u} \times P)+\vec{u} \nabla \times P
$$

where $U$ is the internal energy of fluid per unit mass, $q$ is the heat transferred from the heat source to the unit mass fluid per unit time, $k$ is the thermal conductivity of the fluid, $T$ is the thermodynamic temperature.

The continuity equation is shown in Equation (3)

$$
\frac{\partial \rho}{\partial t}+\frac{\partial\left(\rho u_{x}\right)}{\partial x}+\frac{\partial\left(\rho u_{y}\right)}{\partial y}+\frac{\partial\left(\rho u_{z}\right)}{\partial z}=0
$$

where $u_{x}, u_{y}$ and $u_{z}$ is the component of the velocity $\vec{u}$ salong the $x, y$, and $z$ three coordinate axes.

\section{Meshing and Boundary Condition Setting}

Meshing is the first and most crucial step of CFD simulation calculation. Meshing divides the spatially continuous solution area to obtain many sub-areas and determines the node distribution in each sub-areas. Most of the solution areas, in actual engineering calculations are complex, and the efficiency of CFD calculations and the accuracy of the final results also depend on the divided meshes. Therefore, the division of meshes in irregular areas is an important research field in CFD.

For the symmetrical multi-throttle thrust bearing studied in this article, in the actual meshing process, the overall model size of the bearing can reach tens or even hundreds of millimeters because the thickness of the gas film is on the micron level. The significant difference in size and magnitude leads to errors in the meshing process that are incredibly prone to negative volume and excessive radius of curvature, thereby affecting the final calculation result. Therefore, studying the effect of simulation calculation of symmetrical multi-throttle thrust bearings under different meshing forms is significant in improving the simulation efficiency and calculation accuracy thereof. 
Generally speaking, the meshes used in CFD calculations can be roughly divided into structured and unstructured meshes, as shown in Figure 5. This article will use the following three methods, sliced structured meshes, continuous structured meshes, and unstructured meshes, to divide the mesh and compare the effects on the final calculation.

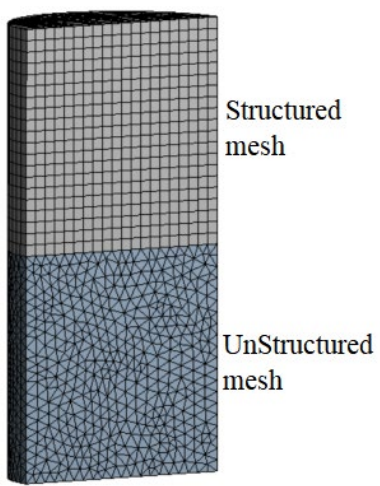

Figure 5. Structured mesh and unstructured mesh.

\subsection{Divide Sliced Structured Meshes}

When meshing the bearing model, is used the slice function to divide different parts of the air film model into independent sub-parts. Moreover, the sweep method and different mesh size parameters are used to divide the mesh separately for different sub-parts. The meshing result and the final mesh quality are shown in Figure 6. The final number of meshed cells is 44,955 , the minimum mesh orthogonal quality is 0.79879 , the maximum mesh orthogonal quality is 1 , the average value is 0.99147 , the maximum skewness is 0.55458 , the minimum skewness is $3.9027 \times 10^{-5}$, and the average value is $6.7402 \times 10^{-2}$. The overall quality of the mesh is good.

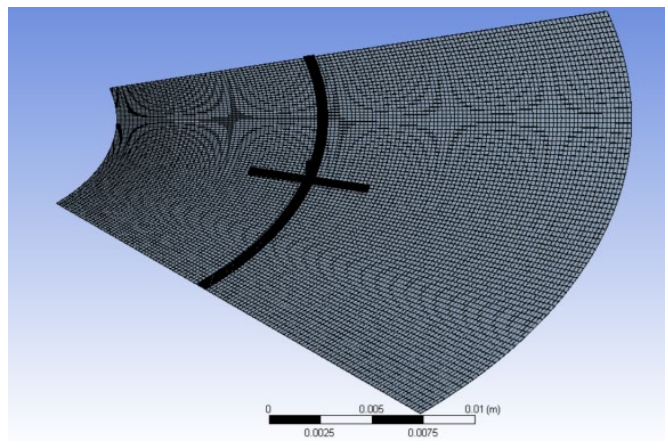

(a)

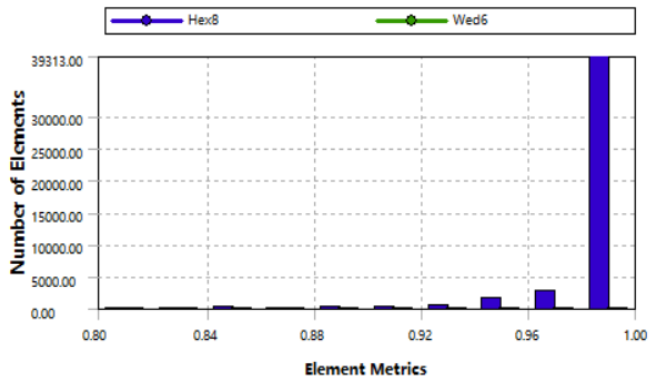

(c)

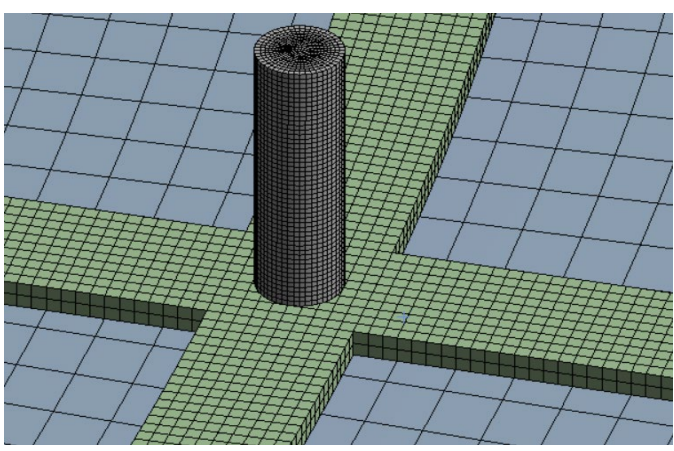

(b)

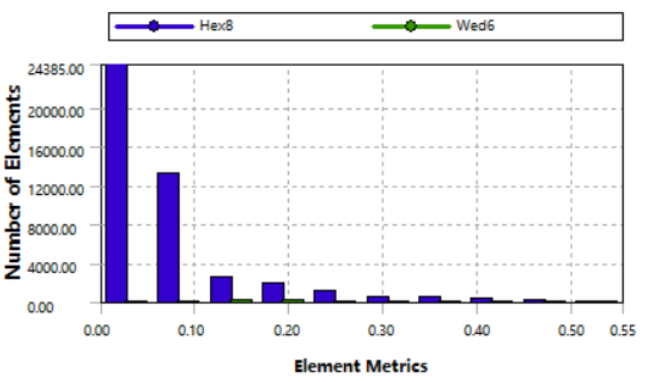

(d)

Figure 6. Mesh division result and division quality: (a) structural overall mesh; (b) local refinement of the mesh at the inlet; (c) mesh orthogonal mass distribution; (d) mesh skewness distribution. 


\subsection{Divide Continuous Structured Meshes}

When divide the continuous structured mesh, the solution area is approached by creating different blocks, and by establishing the mapping relationship between the blocks and the elements of the analysis model such as points, lines, and areas; the mesh of the blocks is mapped to the analysis model. Thereby, a continuous structured mesh of the analysis model is generated. When creating a block, the compound throttle thrust bearing studied in this article can use O-Block mesh nesting for the air film inlet position to obtain a better-quality mesh. The meshing result and the final mesh quality are shown in Figure 7. The final number of meshed cells is 28,485 , and the Jacobian minimum is 0.653 , the Jacobian maximum is 1 , the Erickson skewness minimum is 0.753, and the Erickson skewness maximum is 1 . The overall quality of the mesh is good.

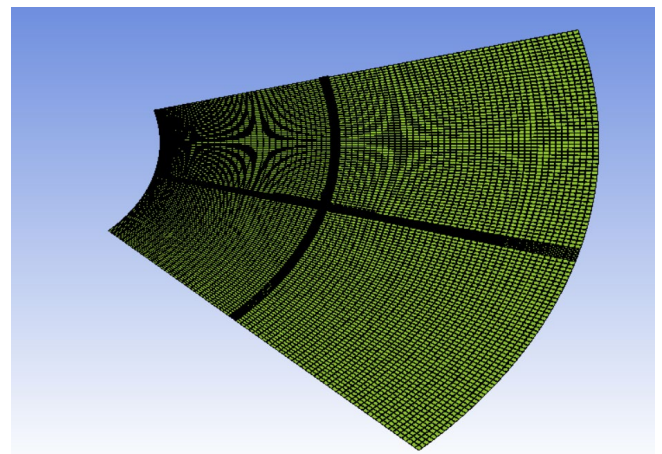

(a)

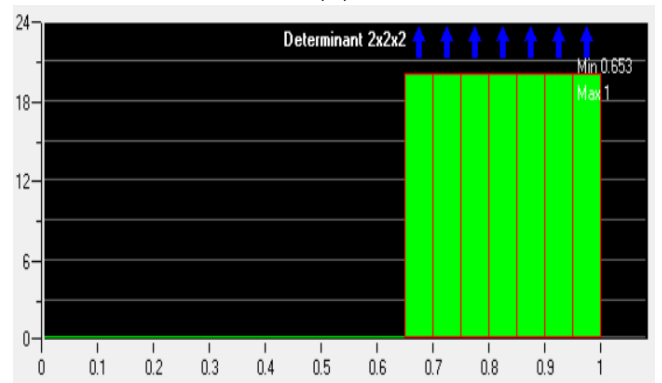

(c)

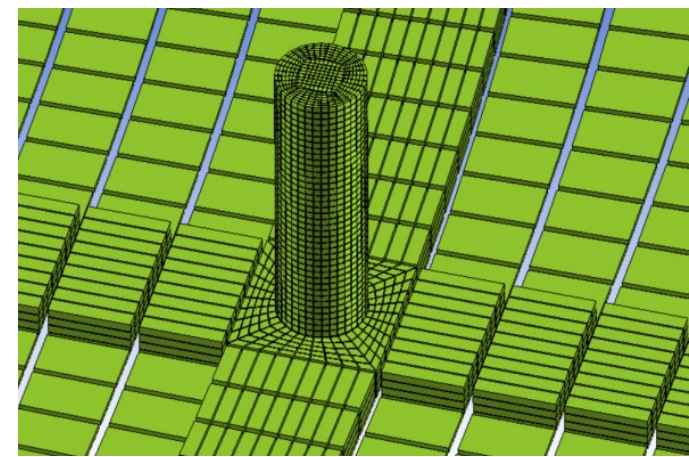

(b)

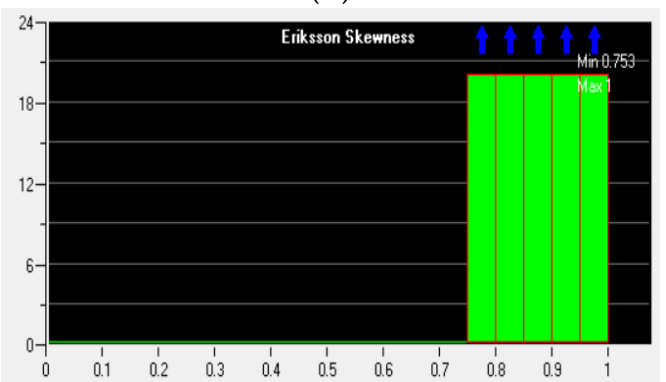

(d)

Figure 7. Mesh division result and division quality: (a) structural overall mesh; (b) local refinement of the mesh at the intake; (c) mesh Jacobian determinant check; (d) mesh Erickson skewness check.

\subsection{Divide Unstructured Meshes}

Since the gas film model differs by tens of thousands of times in the thickness and width directions, if the whole model is directly divided into unstructured meshes, it is straightforward to have a negative volume part, which will affect the simulation analysis and calculation. Therefore, when dividing the unstructured mesh, use the slice function to divide different parts of the air film model into independent sub-parts; the air film part is divided into a structured mesh, and the rest is divided into an unstructured mesh. The meshing result and the final mesh quality are shown in Figure 8. The final number of meshed cells is 115,081 , the minimum mesh orthogonal quality is 0.27569 , the maximum mesh orthogonal quality is 1 , the average value is 0.84881 , the maximum skewness is 0.84691 , the minimum skewness is $1.5881 \times 10^{-6}$, and the average value is 0.24597 . The overall quality of the mesh is good. 


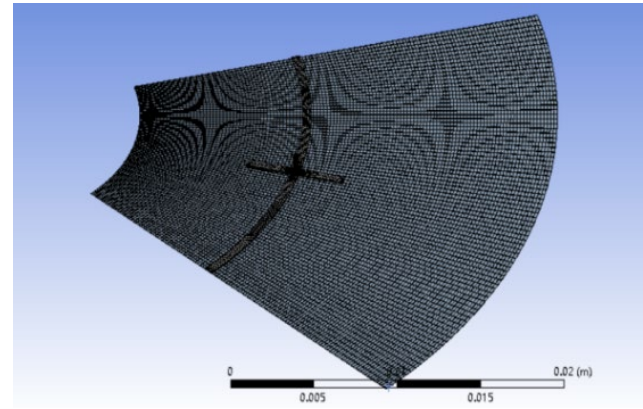

(a)

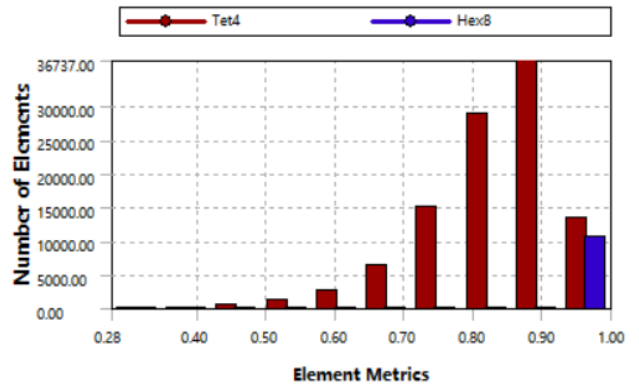

(c)

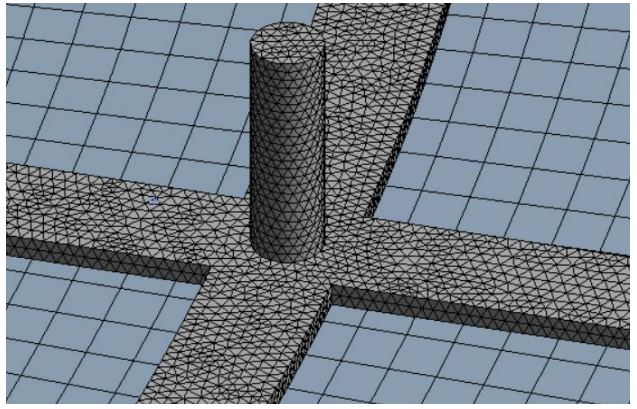

(b)

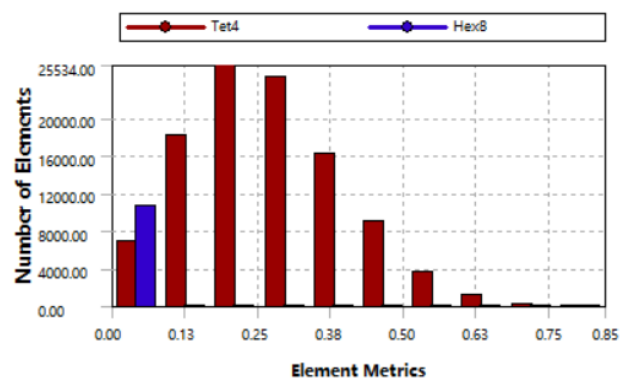

(d)

Figure 8. Mesh division result and division quality: (a) structural overall mesh; (b) local refinement of the mesh at the intake; (c) mesh orthogonal mass distribution; (d) mesh skewness distribution.

\subsection{Boundary Condition Setting}

A total of four boundary conditions are set during simulation analysis:

(1) the air film model material is air, with a density of $1.225 \mathrm{~kg} / \mathrm{m}^{3}$;

(2) the inlet pressure is $0.5 \mathrm{Mpa}$;

(3) the outlet pressure is $0.1 \mathrm{Mpa}$; and

(4) the ambient temperature is $293.15 \mathrm{~K}$.

\section{Comparative Analysis and Comparison of Simulation Results}

4.1. Sliced Structured Mesh

After calculate the flow field, the residual curve is shown in Figure 9a, the model converges after 156 iterative calculation steps, and the cloud charts of pressure distribution of gas film are shown in Figure $8 b$.

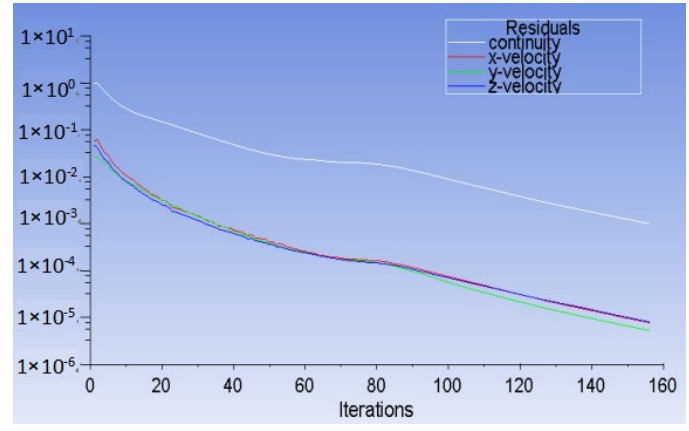

(a)

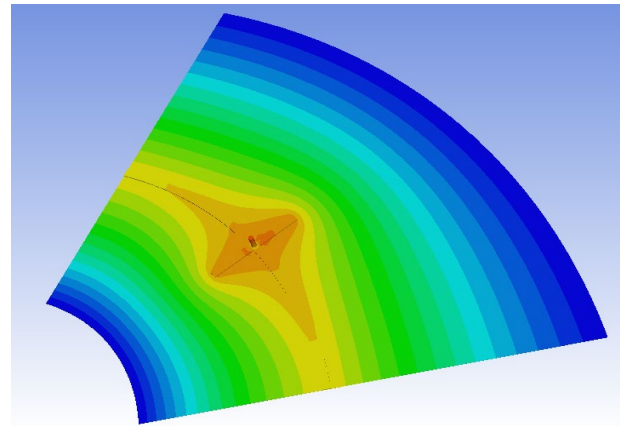

(b)

Figure 9. Analysis results of sliced structured mesh: (a) residual curve; (b) pressure distribution cloud chart. 


\subsection{Continuous Structured Mesh}

After calculate the flow field, the residual curve is shown in Figure 10a, the model is iteratively calculated after 65 steps and the convergence ends, and the cloud charts of pressure distribution of gas film are shown in Figure 10b.

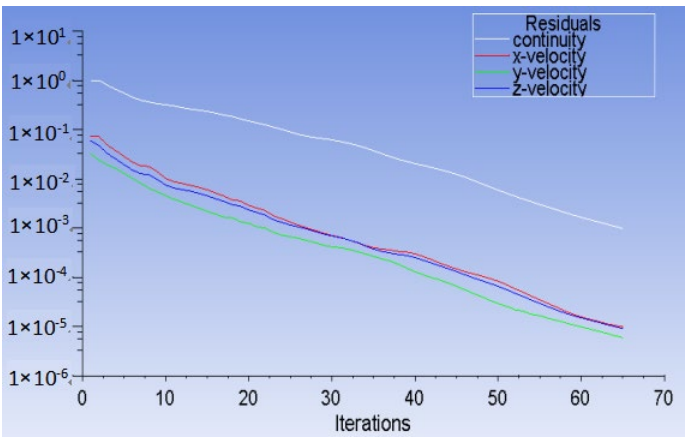

(a)

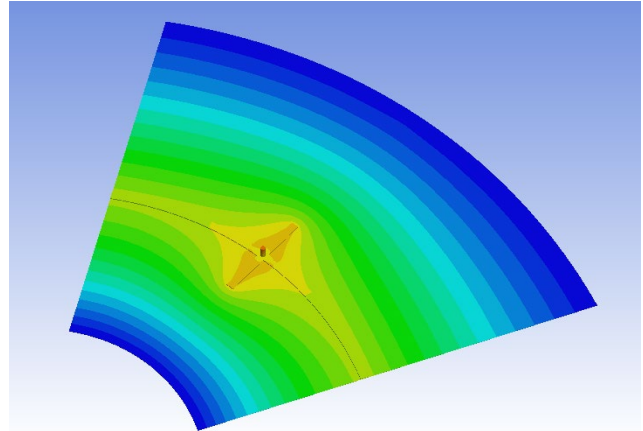

(b)

Figure 10. Analysis results of continuous structured mesh: (a) residual curve; (b) pressure distribution cloud chart.

\subsection{Unstructured Mesh}

After calculate the flow field, the residual curve is shown in Figure 11a, and the model is iterated to about 75 steps, and the curve begins to oscillate. After 300 iterations, calculate and compare the inlet and outlet flow of the model. The result is shown in Figure $11 \mathrm{~b}$. The difference between the inlet and outlet flow rates is $2.738 \times 10^{-6} \mathrm{~kg} / \mathrm{s}$, which only accounts for $0.042 \%$ of the inlet flow rate, so the calculation can be considered to have reached convergence. The cloud charts of pressure distribution of gas film are shown in Figure $11 b$.

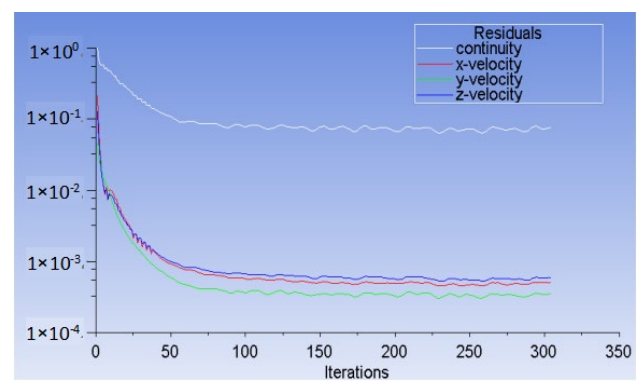

(a)

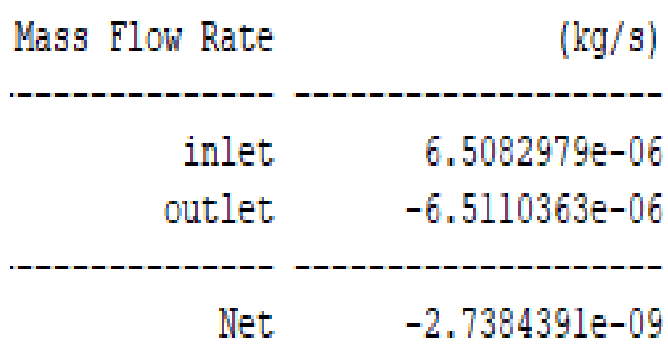

(b)

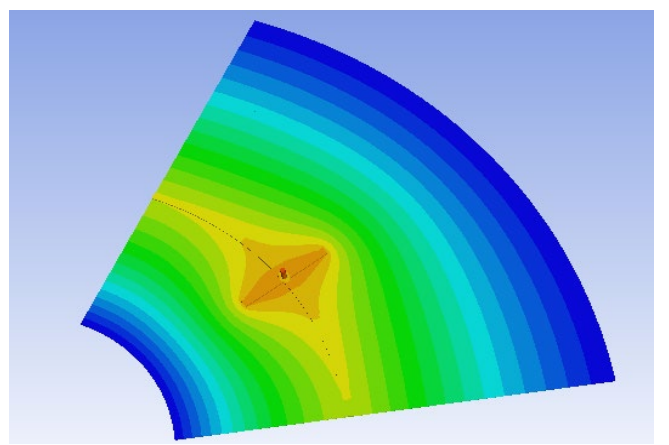

(c)

Figure 11. Unstructured mesh analysis results: (a) residual curve; (b) comparison of import and export flow; (c) pressure distribution cloud chart. 


\subsection{Comparison of Simulation Using Different Meshes}

(1) Quality of the mesh

Mesh quality is an essential indicator for evaluating the results of meshing. Comparing the structured and unstructured meshes that are also sliced and then divided, the number of elements of the structured mesh is 44,955 , which is only $1 / 3$ of the unstructured mesh, but the average orthogonal quality of the mesh is 0.99147 . The average skewness is $6.7402 \times 10^{-2}$, compared with the average orthogonal quality of the unstructured mesh, 0.84881 , and the average skewness, 0.24597 , the mesh quality has improved significantly. By comparing the residual curve calculations, it can be found that the residual curve of the unstructured mesh has the phenomenon of oscillation and non-convergence, while the structured mesh is better at convergence. Thus, it is not hard to obtain structured meshes that can use a smaller number of elements to achieve higher mesh quality.

\section{(2) Computational efficiency}

Obviously, due to the oscillation of the residual curve of unstructured mesh, the computational efficiency has been dramatically affected. For structured mesh, the continuous structured mesh only needs 65 steps to complete the calculation, which is much faster than the 156 steps of the sliced structured mesh. However, one thing that needs to be considered is that using slicing to divide the mesh for more complex structures is much simpler than divide the continuous structured mesh, thereby reducing the pre-processing time. Therefore, we can comprehensively consider these factors when selecting the mesh method.

\section{(3) Calculation accuracy}

The pressure distribution of the three meshes is basically the same at the thrust surface of the bearing. The main difference lies in the pressure distribution near the orifice and throttle groove. For sliced structural mesh, as shown in Figure 12, because the sliced structural mesh is divided separately by the slicing method, the meshes are not continuous at the interface, resulting in an unsmooth pressure gradient at the interface of the orifice and the throttle groove, which results in a particular calculation error.

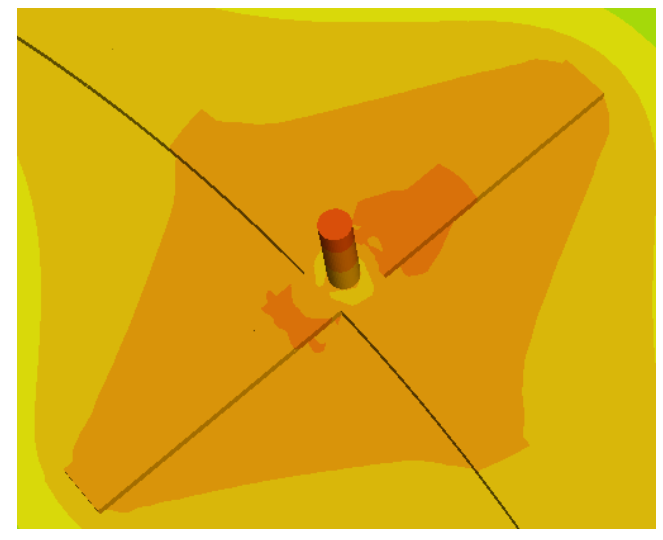

Figure 12. Pressure distribution at the interface of the orifice and the throttle groove.

\section{Influence of Radial Equalizing Groove Parameters}

Based on the above research, this section uses continuous structural mesh when meshing the bearing and further studies the influence of the size of the radial equalizing groove on the bearing capacity of a symmetrical multi-throttle thrust bearing. The comparative study analyzes the degree of influence of radial equalizing groove length $L_{\mathrm{g}}$, radial equalizing groove width $\mathrm{bg}$, radial equalizing groove depth $\mathrm{hg}$, and gas film thickness $\mathrm{h}$ on bearing capacity $W$. Different radial equalizing groove size parameters are set between each group. In contrast, the other bearing parameters are unchanged. The setting dimensions are as follows: bearing inner radius $R_{2}=10 \mathrm{~mm}$, outer radius $R_{1}=35 \mathrm{~mm}$, orifice distribution 
circle radius $R_{3}=20 \mathrm{~mm}$, orifice diameter $d=0.3 \mathrm{~mm}$, the number of orifices is 6 , and the orifice air supply pressure $0.5 \mathrm{MPa}$.

\subsection{The Influence of Radial Equalizing Groove Length on Bearing Capacity}

Three simulation experiments are set up according to the gas film thickness $h=8 \mu \mathrm{m}$, $9 \mu \mathrm{m}$, and $10 \mu \mathrm{m}$, respectively. In each set of simulations, the radial equalizing groove width $b_{\mathrm{g}}$ is $0.6 \mathrm{~mm}$ and the radial equalizing groove depth $h_{\mathrm{g}}$ is $0.05 \mathrm{~mm}$. The lengths of the radial equalizing groove in each group are respectively set to $L_{\mathrm{g}}=4 \mathrm{~mm}, 6 \mathrm{~mm}, 8 \mathrm{~mm}$, $10 \mathrm{~mm}$, and $12 \mathrm{~mm}$. According to the simulation results, the relationship between the radial groove length and the bearing capacity is drawn, as shown in Figure 13. It can be seen from the graph that as the air film thickness $h$ decreases, the bearing capacity increases, and the air film pressure increases rapidly. Therefore, the smaller the air film gap, the higher the bearing capacity; at the same time, as the length $L_{\mathrm{g}}$ of the radial equalizing groove increases, the bearing capacity also gradually increases, and as the length $L_{\mathrm{g}}$ of the radial equalizing groove increases, the increasing trend of the bearing capacity becomes slower.

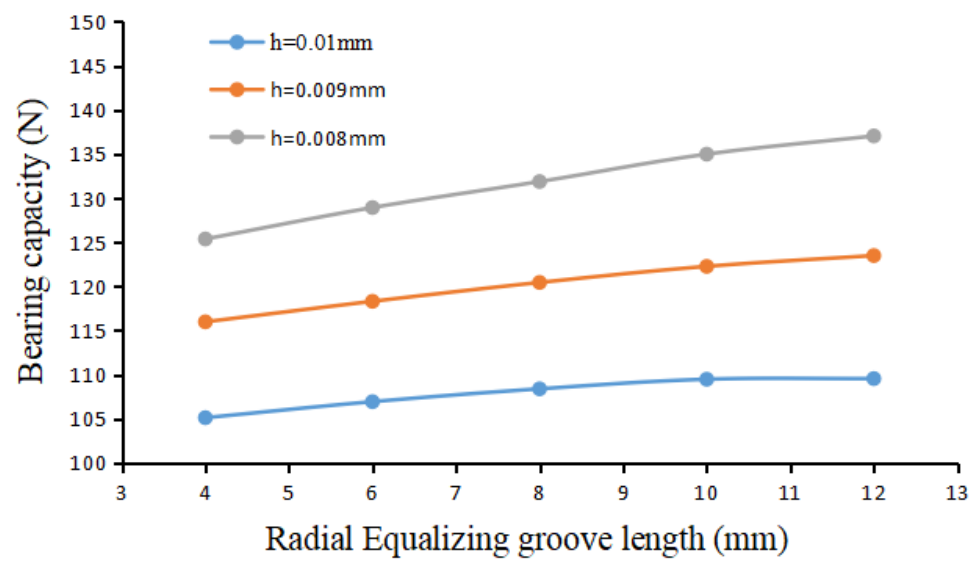

Figure 13. The influence of radial equalizing groove length on bearing capacity.

Figure 14 shows the cloud diagram of gas film pressure distribution under radial equalizing grooves of different lengths. It can be seen from the figure that as the length $L_{\mathrm{g}}$ of the radial equalizing groove increases, the area of the high-pressure zone is gradually increasing. When the length of the radial equalizing groove $L_{\mathrm{g}}$ is short, the high-pressure area of the gas film is only concentrated in the vicinity of the radial equalizing groove. As the groove length $L_{\mathrm{g}}$ increases, the high-pressure zone of the gas film spreads radially and axially with the orifice as the center. However, when the groove length $L_{\mathrm{g}}$ is too long because the two ends of the radial equalizing groove are too close to the inner and outer diameter edges of the bearing, the pressure at the end of the radial equalizing groove decreases, and the pressure at the same radius on the radial equalizing groove increases with the groove length $L_{\mathrm{g}}$. Instead, it decreases. Therefore, under the combined influence of the above factors, the air film bearing capacity $W$ and the length of the radial equalizing groove $L_{\mathrm{g}}$ show that, as the length of the radial equalizing groove $L_{\mathrm{g}}$ increases, the bearing capacity $W$ also gradually increases, and as the length of the radial equalizing groove $L_{g}$ increases, the load-bearing capacity $W$ increases and the trend becomes slower.

The length $L_{\mathrm{g}}$ of the radial equalizing groove should not be too large in the design. In the design, it is generally necessary to ensure that the total volume of the air cavity occupies between $2 \%$ and $10 \%$ of the total volume of the air film to ensure the stability of the bearing thereby. When the length $L_{\mathrm{g}}$ of the radial equalizing groove is too large, it is easier to produce self-excited vibration of the air hammer. 

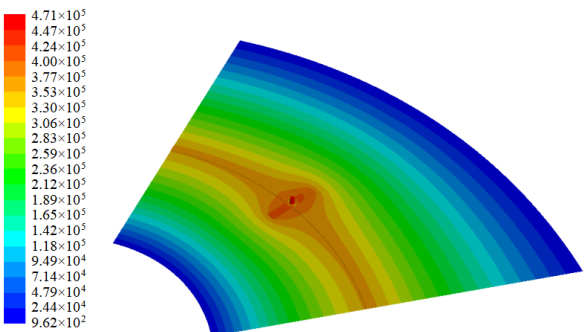

(a)
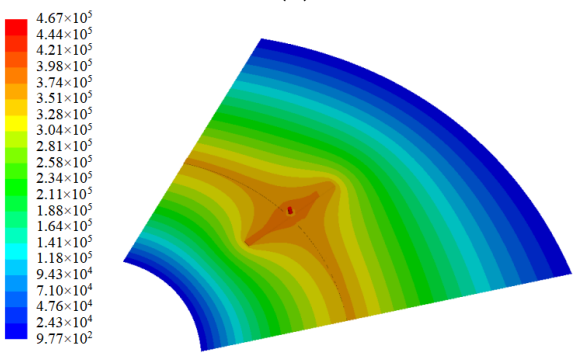

(c)
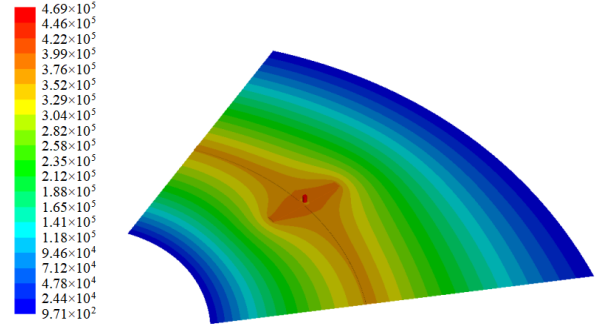

(b)
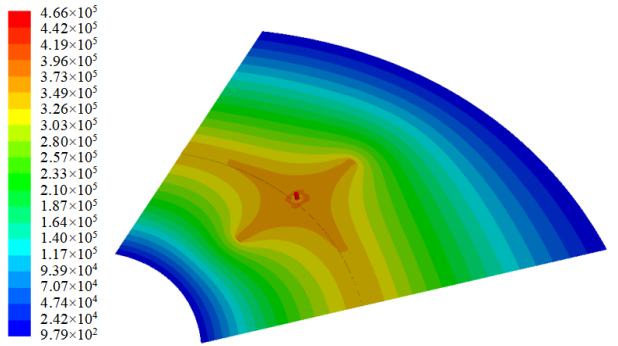

(d)

Figure 14. Cloud diagram of gas film pressure distribution under pressure radial equalizing grooves of different lengths: (a) length of radial equalizing groove $L_{\mathrm{g}}=4 \mathrm{~mm}$; (b) length of radial equalizing groove $L_{\mathrm{g}}=6 \mathrm{~mm}$; (c) length of radial equalizing groove $L_{\mathrm{g}}=8 \mathrm{~mm}$; (d) length of radial equalizing groove $L_{\mathrm{g}}=10 \mathrm{~mm}$.

\subsection{The Influence of Radial Equalizing Groove Width on Bearing Capacity}

According to the gas film thickness $h=8 \mu \mathrm{m}, 9 \mu \mathrm{m}, 10 \mu \mathrm{m}$, three simulation experiments are prepared, respectively. In each set of simulations, the radial equalizing groove length $L_{\mathrm{g}}$ is $10 \mathrm{~mm}$, and the radial equalizing groove depth $h_{\mathrm{g}}$ is $0.05 \mathrm{~mm}$. The widths of the radial equalizing groove in each group are respectively set as $b_{\mathrm{g}}=0.4 \mathrm{~mm}, 0.5 \mathrm{~mm}$, $0.6 \mathrm{~mm}, 0.7 \mathrm{~mm}, 0.8 \mathrm{~mm}$. According to the simulation results, the relationship between the radial groove width $b_{\mathrm{g}}$ and the bearing capacity $W$ is drawn, as shown in Figure 15. It can be seen from the graph that as the groove width $b_{\mathrm{g}}$ of the radial equalizing groove increases, the bearing capacity of the bearing increases correspondingly, but the influence of the groove width $b_{\mathrm{g}}$ of the radial equalizing groove on the bearing capacity is minimal. When the gas film thickness $h$ is $10 \mu \mathrm{m}$, the difference between the bearing capacity of a bearing with a groove width $b_{\mathrm{g}}$ of $0.8 \mathrm{~mm}$ and the bearing capacity $W$ of a bearing with groove width $b_{\mathrm{g}}$ of $0.4 \mathrm{~mm}$ is only $1.5 \mathrm{~N}$. In consideration of reducing the volume of the air cavity to ensure the stability of the bearing, a minor groove width $b_{\mathrm{g}}$ can be selected in the design.

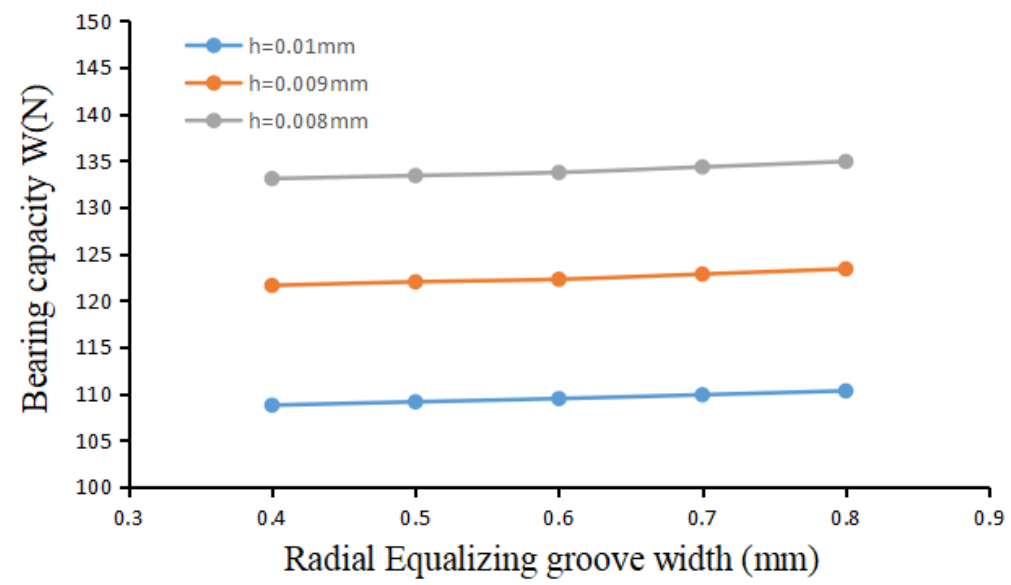

Figure 15. The influence of radial equalizing groove width on bearing capacity. 


\subsection{The Influence of Radial Equalizing Groove Depth on the Bearing Capacity}

According to the gas film thickness $h=8 \mu \mathrm{m}, 9 \mu \mathrm{m}, 10 \mu \mathrm{m}$, three simulation experiments are prepared, respectively. In each set of simulations, the radial equalizing groove length $L_{\mathrm{g}}$ is $10 \mathrm{~mm}$, and the radial equalizing groove depth $h_{\mathrm{g}}$ is $0.05 \mathrm{~mm}$. The depths of the radial equalizing groove in each group are respectively set as $h_{\mathrm{g}}=40 \mu \mathrm{m}, 50 \mu \mathrm{m}$, $60 \mu \mathrm{m}, 70 \mu \mathrm{m}, 80 \mu \mathrm{m}$. According to the simulation results, the relationship between the radial groove depth $h_{\mathrm{g}}$ and the bearing capacity $W$ is drawn, as shown in Figure 16. It can be seen from the graph that as the depth $h_{\mathrm{g}}$ of the radial equalizing groove increases, the bearing capacity $W$ of the bearing increases correspondingly. When the thickness $h$ of the air film is smaller, the increase in the depth $h_{\mathrm{g}}$ of the radial equalizing groove has a more significant influence on the bearing capacity $W$. This is because the air film gap is small, and the difference in $h$ between the depth $h_{\mathrm{g}}$ of the radial equalizing groove and the air film thickness becomes more prominent, and the effect of surface throttling is relatively apparent. When the air film thickness $h=10 \mu \mathrm{m}$, as the bearing groove depth $h_{\mathrm{g}}$ increases from $40 \mu \mathrm{m}$ to $80 \mu \mathrm{m}$, the bearing capacity increases by $10.59 \mathrm{~N}$. When the air film thickness $h=8 \mu \mathrm{m}$, the bearing capacity increment reaches $16.28 \mathrm{~N}$, about 1.5 times higher than when the air film thickness $h=10 \mu \mathrm{m}$.

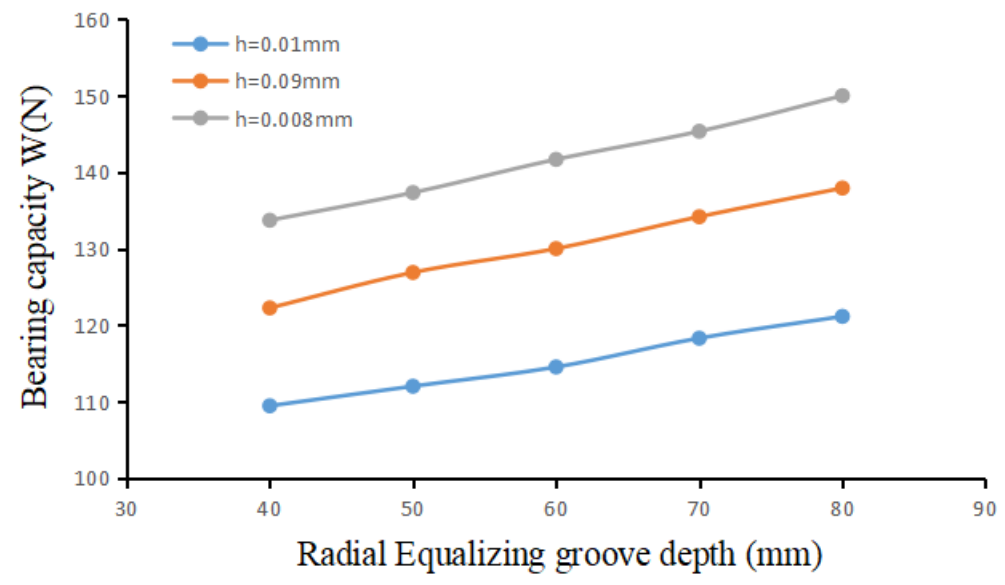

Figure 16. The influence of radial equalizing groove depth on the bearing capacity.

\subsection{Verifications}

In Ref. [18], the researchers conducted an experimental analysis of the static characteristics of a thrust bearing with circumferential pressure equalizing grooves and orifices and plotted the load-carrying capacity curves under different gas film thicknesses. Compared with reference [18], the thrust bearing structure studied in this paper only adds radial pressure equalizing grooves, and the rest of the structure is the same, so that it can be directly compared with the experimental data in Ref. [18].

Since the thickness of the gas film studied in this paper ranges from $8 \mu \mathrm{m}$ to $10 \mu \mathrm{m}$, the gas film thicknesses are selected as $5 \mu \mathrm{m}, 6 \mu \mathrm{m}, 7 \mu \mathrm{m}, 8 \mu \mathrm{m}, 9 \mu \mathrm{m}$, and $10 \mu \mathrm{m}$ in the comparison and verification, and the rest of the structural parameters are set according to reference [18]. During the simulation, the smallest symmetrical part of the gas film is taken again and continuous structural mesh is used when meshing the bearing. Figure 17 compares the simulation results with the experimental results in Ref. [18]. It is not difficult to see that the simulation results are in good agreement with the experiments, which proves that the simulation analysis method and calculation results in this paper have high accuracy. 


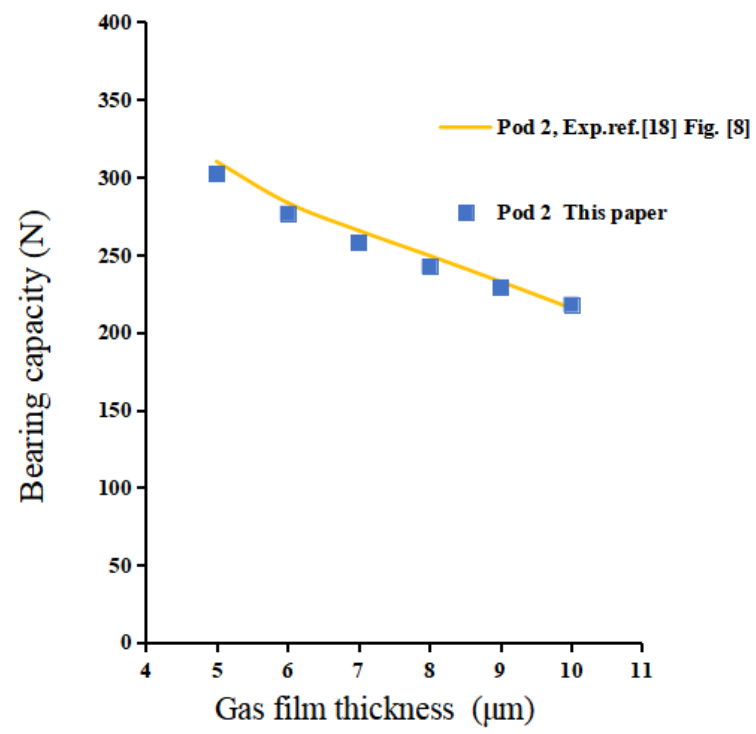

Figure 17. The comparison results of bearing capacity between simulation and previous.

\section{Conclusions}

The paper takes symmetrical multi-throttle thrust bearing as the research object, uses three methods to divide the mesh, and conducts simulation comparison analyses. The analyses' results show that the unstructured mesh will oscillate and fail to converge in calculating the residual curve, and the sliced structural mesh is divided simply, but its calculation speed and accuracy are slightly inferior to the continuous structure grid. On this basis, a continuous structural mesh is used to divide the bearing, and the influence of the radial throttle groove on the pressure distribution and static characteristics of the bearing is studied. The analyses' results show that the thickness of the air film $h$ has the most significant influence on the bearing capacity, the length Lg and depth hg of the throttle groove also have a significant influence on the bearing capacity, while the width of the throttle groove has a weak influence on the bearing capacity. Thus, at a particular air cavity volume, priority should be given to reducing the width of the throttle groove and increasing the length and depth of the throttle groove to improve the bearing capacity. These works guide the design and simulation of symmetrical multi-throttle thrust bearings.

Author Contributions: Conceptualization, Y.L.; writing-original draft preparation, Z.C.; methodology D.W.; validation, Y.Z.; investigation, X.W.; supervision, C.S. All authors have read and agreed to the published version of the manuscript.

Funding: This research was funded by the National Natural Science Foundation major research projects of China (grant number 91960109), the National Natural Science Foundation of China (grant number 51805117), the China postal Postdoctoral Science Foundation (grant number 2019M651279), the Heilongjiang Postdoctoral Fund (grant number LBH-Z18078), the scientific and technological cooperation project of Heilongjiang Provincial Academy of Sciences (grant number YS19A15).

Institutional Review Board Statement: Not applicable.

Informed Consent Statement: Not applicable.

Data Availability Statement: Not applicable.

Acknowledgments: The author thanks the National Natural Science Foundation major research projects of China (grant number 91960109), the National Natural Science Foundation of China (grant number 51805117), the China postal Postdoctoral Science Foundation (grant number 2019M651279), the Heilongjiang Postdoctoral Fund (grant number LBH-Z18078), the scientific and technological cooperation project of Heilongjiang Provincial Academy of Sciences (grant number YS19A15)for funding and the experimental equipment support from Center of Ultra-precision Optoelectronic Instrument Engineering. 
Conflicts of Interest: The authors declare no conflict of interest.

\section{References}

1. Wu, L.; Leng, J.; Ju, B. Digital Twins-Based Smart Design and Control of Ultra-Precision Machining: A Review. Symmetry 2021, 13, 1717. [CrossRef]

2. Li, Z.; Shi, Y. Multi-Objective Parameter Optimization for Disc Milling Process of Titanium Alloy Blisk Channels. Symmetry 2019, 11, 173. [CrossRef]

3. Sarhan, A. Investigate the spindle errors motions from thermal change for high-precision CNC machining capability. Int. J. Adv. Manuf. Technol. 2014, 70, 957-963. [CrossRef]

4. Zha, J.; Chen, Y.; Zhang, P. Precision design of hydrostatic thrust bearing in rotary table and spindle. Proc. Inst. Mech. Eng. Part B-J. Eng. Manuf. 2018, 232, 2044-2053. [CrossRef]

5. Fang, C.; Huo, D.; Huang, X. A comprehensive analysis of factors affecting the accuracy of the precision hydrostatic spindle with mid-thrust bearing layout. Int. J. Adv. Manuf. Technol. 2021, 10, 949-967. [CrossRef]

6. Zhao, B.; Wang, L.; Tan, J.; Sun, C. Aircraft Engine Rotor Assembly Method and Device. Patent WO2015120749 A1, 7 August 2017.

7. De Santis, M.; Silvestri, L.; Forcina, A.; Di Bona, G.; Di Fazio, A.R. Preliminary Realization of an Electric-Powered Hydraulic Pump System for a Waste Compactor Truck and a Techno-Economic Analysis. Appl. Sci. 2021, 11, 3033. [CrossRef]

8. Sahto, M.; Wei, W.; He, L.; Imran, M.; Gong, W. Modelling and Simulation of Aerostatic Thrust Bearings. IEEE Access 2020, 99, 121299-121310. [CrossRef]

9. Yan, R.; Wang, L.; Wang, S. Investigating the influences of pressure-equalizing grooves on characteristics of aerostatic bearings based on CFD. Ind. Lubr. Tribol. 2019, 71, 4. [CrossRef]

10. Gao, S.; Shi, Y.; Xu, L.; Chen, H.; Cheng, K. Investigation on influences of herringbone grooves for the aerostatic journal bearings applied to ultra-high-speed spindles. Proc. Inst. Mech. Eng. Part C-J. Eng. Mech. Eng. Sci. 2019, 16, 5795-5812. [CrossRef]

11. Cui, H.; Wang, Y.; Wang, B.; Yang, H.; Xia, H. Numerical Simulation and Experimental Verification of the Stiffness and Stability of Thrust Pad Aerostatic Bearings. Chin. J. Mech. Eng. 2018, 02, 186-197. [CrossRef]

12. Zhou, Y.; Chen, X.; Chen, H. A hybrid approach to the numerical solution of air flow field in aerostatic thrust bearings. Tribol. Int. 2016, 102, 444-453. [CrossRef]

13. Ma, W.; Cui, J.; Liu, Y.; Tan, J. Improving the pneumatic hammer stability of aerostatic thrust bearing with recess using damping orifices. Tribol. Int. 2016, 103, 281-288. [CrossRef]

14. Yadav, S.; Rajput, A.; Ram, N.; Sharma, S.; Gachot, C. A novel technique to compute static and dynamic performance characteristics of aerostatic thrust bearing. Ind. Lubr. Tribol. 2017, 70, 84-96. [CrossRef]

15. Müller, C.; Greco, S.; Kirsch, B.; Aurich, J. A Finite Element Analysis of Air Bearings Applied in Compact Air Bearing Spindles. Procedia CIRP 2017, 58, 607-612. [CrossRef]

16. Chang, S.; Chan, C.; Jeng, Y. Numerical analysis of discharge coefficients in aerostatic bearings with orifice-type restrictors. Tribol. Int. 2015, 90, 157-163. [CrossRef]

17. Wei, L.; Ruibo, Y.; Jing, L. Computational Fluid Dynamics of aerostatic bearings with the finite volume method. In Proceedings of the 2011 IEEE 5th International Conference on Robotics, Automation and Mechatronics (RAM), Qingdao, China, 17-19 September 2011; pp. 270-274. [CrossRef]

18. Belforte, G.; Colombo, F.; Raparelli, T.; Trivella, A.; Viktorov, V. Comparison between grooved and plane aerostatic thrust bearings: Static performance. Meccanica 2011, 46, 547-555. [CrossRef] 